\title{
TRANSCENDENTAL $\ell$-ADIC GALOIS REPRESENTATIONS
}

\author{
Chandrashekhar Khare, Michael Larsen, and Ravi Ramakrishna
}

\section{Introduction}

Let $F$ be a number field and $G_{F}=\operatorname{Gal}(\bar{F} / F)$ be its absolute Galois group. Let $\mathbb{C}_{\ell}$ be the completion of $\overline{\mathbb{Q}}_{\ell}$. In this paper we study continuous $\ell$-adic Galois representations $\rho: G_{F} \rightarrow \mathrm{GL}_{n}\left(\mathbb{C}_{\ell}\right)$. We call such representations transcendental when they do not have models over a finite extension of $\mathbb{Q}_{\ell}$. Continuous $\ell$-adic representations of $G_{F}$ which arise classically (for example from geometry) have models over a finite extension of $\mathbb{Q}_{\ell}$. By an argument that is part of the "folklore", one does not get any "new representations" if one considers continuous representations $\rho: G_{F} \rightarrow \mathrm{GL}_{n}\left(\overline{\mathbb{Q}}_{\ell}\right)$ as these have models over some finite extension $K$ of $\mathbb{Q}_{\ell}$. This follows simply from the fact that a compact subgroup $C$ of $\mathrm{GL}_{n}\left(\overline{\mathbb{Q}}_{\ell}\right)$ in fact lies in $\mathrm{GL}_{n}(K)$ for $K$ a sufficiently large finite extension of $\mathbb{Q}_{\ell}$ (Baire category theorem: namely by this we know that for some finite extension $K^{\prime} / \mathbb{Q}_{\ell}$ the intersection of $C$ and $\mathrm{GL}_{n}\left(K^{\prime}\right)$ is an open, and hence finite index, subgroup of $C$ ).

We describe below (see Theorem 4.1) the construction of a transcendental representation, which

- is semisimple, and therefore unramified at a density one set of places by Theorem 2.5 below,

- at a density one set of unramified places has characteristic polynomials of Frobenii defined over $\overline{\mathbb{Q}}_{\ell}$,

- does not have a model over $\overline{\mathbb{Q}}_{\ell}$, or equivalently over any finite extension of $\mathbb{Q}_{\ell}$.

By another result we prove below (see Corollary 3.2), such representations are necessarily infinitely ramified. In the course of this paragraph, we have also given a description of the three main results of this work.

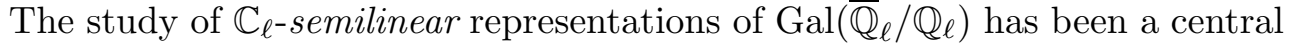
object of study in the subject of $p$-adic (or $\ell$-adic in present notation!) Hodge theory for more than 30 years. The $\mathbb{C}_{\ell}$-linear representations of global Galois groups that we examine here have to our knowledge not been studied hitherto.

Received by the editors August 15, 2004.

Khare is partially supported by NSF Grant DMS-0355528.

Larsen is partially supported by NSF Grant DMS-0100537.

Ramakrishna is partially supported by NSF Grants DMS-0102173, DMS-0400232, and the AMS Centennial Research Fellowship. 
The rationale that we can offer for the representations considered here, besides the obvious one that we find them diverting, may be summarised as follows.

1. Transcendental $\ell$-adic representations include the study of big Galois representations, that have been studied earlier in the work of Hida, Mazur et al. as one has continuous embeddings $\mathbb{Z}_{\ell}\left[\left[X_{1}, \ldots, X_{r}\right]\right] \hookrightarrow \mathbb{C}_{\ell}$ (for arbitrary $r)$.

2. As the theory of complex representations of various kinds of groups illustrates, it is natural to study representations over complete, algebraically closed fields that are further minimal. Here by minimal we mean in the sense of $\mathbb{C}$ being a complete algebraic closure of $\mathbb{R}$, the $\infty$-adic completion of its prime field $\mathbb{Q}$, or $\mathbb{C}_{\ell}$ being the completion of an algebraic closure of $\mathbb{Q}_{\ell}$, the $\ell$-adic completion of its prime field $\mathbb{Q}$.

3. This is a continuation of the point above. Transcendental Galois representations with values in $\mathrm{GL}_{n}\left(\mathbb{C}_{\ell}\right)$ can arise as limits of representations that are all defined over $\overline{\mathbb{Q}}_{\ell}$.

\section{Cebotarev density and ramification}

In this section, we consider continuous Galois representations taking values in the characteristic-zero field of fractions $K$ of a valuation ring $V$. (We do not assume that $V$ is discrete, complete, has finite residue field, or has valaution of rank-one.) Our goal is to prove that continuous semisimple Galois representations taking values in $K$ are unramified outside a thin set of primes. This was shown in the locally compact case in [Kh-Raj]. We follow the basic strategy of [Kh-Raj]. A crucial part of that paper, the estimate of volumes of "tubular neighborhoods" of subvarieties (following J-P. Serre [S2]), needs to be replaced by a softer technique. Our method does not give the Hausdorff dimension as in the paper of Serre. It does however allow us to prove that the measure of the intersection of a compact subgroup $\Gamma$ of $\mathrm{GL}_{n}(K)$ with a sufficiently small tubular neighborhood of a codimension $\geq 1$ subvariety of the Zariski closure of $\Gamma$ can be made as small as desired. This also proves a Cebotarev density theorem for transcendental $\ell$-adic representations (see Theorem 2.4) that might be of independent interest.

For the convenience of the reader, we begin by recalling that the standard argument for finding an integral basis for representations of compact groups does not depend on the compactness of $V$.

Lemma 2.1. Every finitely generated torsion-free module over a valuation ring is free.

Proof. Let $V$ be a valuation ring with valuation $v$ and $M$ a finitely generated torsion-free $V$-module. Let $\left\{m_{1}, m_{2}, \ldots, m_{k}\right\}$ be a minimal set of generators of $M$. We claim they form a basis. If not, after reindexing there exists a linear combination $a_{1} m_{1}+\cdots+a_{r} m_{r}=0$, where $1 \leq r \leq k$, all $a_{i}$ are non-zero, and 


$$
\begin{aligned}
& v\left(a_{1}\right) \geq v\left(a_{2}\right) \geq \cdots \geq v\left(a_{r}\right) \text {. Then, as } M \text { is torsion-free, } \\
& \qquad m_{r}=-\frac{a_{1}}{a_{r}} m_{1}-\cdots-\frac{a_{r-1}}{a_{r}} m_{r-1} \in V m_{1}+\cdots+V m_{r-1},
\end{aligned}
$$

contrary to the minimality of $\left\{m_{i}\right\}$.

Lemma 2.2. Let $V$ be a valuation ring with fraction field $K, n$ a positive integer, and $\Gamma$ a compact subgroup of $\mathrm{GL}_{n}(K)$. Then $\Gamma$ can be conjugated within $\mathrm{GL}_{n}(K)$ into a subgroup of $\mathrm{GL}_{n}(V)$.

Proof. Let $\Gamma^{\circ}=\Gamma \cap \mathrm{GL}_{n}(V)$. Then $\Gamma^{\circ}$ is an open subgroup of $\Gamma$ and therefore of finite index. Consider the coset decomposition $\Gamma=\gamma_{1} \Gamma^{\circ} \cup \cdots \cup \gamma_{m} \Gamma^{\circ}$. Let $M=\gamma_{1} V^{n}+\cdots+\gamma_{m} V^{n}$. Then $M$ is a finitely generated $V$-submodule of $K^{n}$ (which is torsion-free). Therefore it is torsion-free, and as it spans $K^{n}$, it is isomorphic to $V^{n}$. It follows that there exists an element of $\mathrm{GL}_{n}(K)$ mapping the original basis of $V^{n}$ to a basis of $M$.

2.1. Cebotarev density. Our goal is to show that Zariski-closed subsets of positive codimension in the Zariski closure of the image of a Galois representation capture only a density-zero set of Frobenius elements. We do this by considering "tubular neighborhoods" of such closed subsets and showing that as "radius" goes to 0 , measure goes to 0 as well. The following proposition is the key.

Proposition 2.3. Let $\Gamma$ denote a compact subgroup of $\mathrm{GL}_{n}(K)$, $\mu$ a Haar measure on $\Gamma$ normalised so that $\mu(\Gamma)=1, G$ the Zariski closure of $\Gamma$ in $\mathrm{GL}_{n}$, and $f$ an element of the coordinate ring of $\mathrm{GL}_{n}$ over $K$ which does not vanish identically on any component of $G$. Then for all $\epsilon>0$, there exists $\alpha$ in the value group of $V$ such that

$$
\mu(\{\gamma \in \Gamma \mid v(f(\gamma))>\alpha\})<\epsilon .
$$

Proof. Our assumption that $K$ is characteristic-zero implies $G$ is smooth and thus: (i) its connected components are its irreducible components, and (ii) the formation of $G$ commutes with extension of the base field (such as to an algebraic closure of $K$ ). Thus we may assume $K$ to be algebraically closed (we owe this reduction to the referee).

We call a (Zariski)-closed subset $X \subset G$ thin if for every finite subset $\left\{f_{1}, \ldots, f_{k}\right\}$ of the coordinate ring $A$ of $\mathrm{GL}_{n}$ such that $Z\left(f_{1}, \ldots, f_{k}\right)=X$, where $Z\left(f_{1}, \ldots, f_{k}\right)$ denotes the zero locus of $f_{1}, \cdots, f_{k}$, we have

$$
\lim _{\alpha \rightarrow \infty} \mu\left(\left\{\gamma \in \Gamma \mid \forall i, v\left(f_{i}(\gamma)\right)>\alpha\right\}\right)=0 .
$$

The proposition follows immediately from the more general statement that every closed subset $X$ of $G$ of codimension $\geq 1$ is thin. We prove this by Noetherian induction. The induction step consists in proving that $X$ is thin if all of its proper closed subsets are thin.

We consider first the case when $X$ is the empty set. Note that $X$ is empty if and only if $\left(f_{1}, \ldots, f_{k}\right)=A$. This happens if and only if there exist $a_{1}, \ldots, a_{k} \in$ 
$A$ such that

$$
a_{1} f_{1}+\cdots+a_{k} f_{k}=1 \text {. }
$$

In particular

$$
a_{1}(\gamma) f_{1}(\gamma)+\cdots+a_{k}(\gamma) f_{k}(\gamma)=1
$$

for all $\gamma \in \Gamma$. Applying $v$, we obtain

$$
\min _{1 \leq i \leq k} v\left(a_{i}(\gamma)\right)+v\left(f_{i}(\gamma)\right) \leq 0
$$

On the other hand, the sets

$$
\Gamma_{i, \alpha}:=\left\{\gamma \in \Gamma \mid v\left(a_{i}(\gamma)\right) \geq-\alpha\right\}
$$

are open, so by compactness, there exists $\alpha_{0}$ in the value group of $V$ with $v\left(a_{i}(\gamma)\right) \geq-\alpha_{0}$ for all $i$, and for all $\gamma \in \Gamma$. Thus,

$$
\left\{\gamma \in \Gamma \mid \forall i, v\left(f_{i}(\gamma)\right)>\alpha_{0}\right\}=\emptyset .
$$

The base case for our induction has been established.

For the induction step, note that $\Gamma$ acts on $\mathrm{GL}_{n}$ by left-translation and therefore acts on $A$. We write $f^{\gamma}$ for the image of $f \in A$ under left translation by a $\gamma \in \Gamma$. For $x \in X$ and $\gamma_{1}, \ldots, \gamma_{i} \in \Gamma$, the closed subset

$$
\left\{g \in G \mid g x \in \gamma_{1} X \cup \cdots \cup \gamma_{i} X\right\}
$$

of $G$ has dimension $\operatorname{dim} X<\operatorname{dim} G$. As $\Gamma$ is dense in $G$, there exists an infinite sequence $\gamma_{1}, \gamma_{2}, \ldots \in \Gamma$ such that $\gamma_{i} X \neq \gamma_{j} X$ for all $i \neq j$. By the induction hypothesis, $\gamma_{j}^{-1} \gamma_{i} X \cap X$ is thin for all $i \neq j$. Recall that $\left(f_{1}, \ldots, f_{k}\right)$ is the ideal in $A$ such that $Z\left(f_{1}, \ldots, f_{k}\right)=X$. Then

$$
Z\left(f_{1}, \ldots, f_{k}, f_{1}^{\gamma_{j}^{-1} \gamma_{i}}, \ldots, f_{k}^{\gamma_{j}^{-1} \gamma_{i}}\right)=\gamma_{j}^{-1} \gamma_{i} X \cap X
$$

so for any given positive integer $n$ there exists $\alpha$ (that depends on $n$ ) such that

$$
\mu\left(\left\{\gamma \in \Gamma \mid \forall h \leq k, v\left(f_{h}(\gamma)\right)>\alpha, v\left(f_{h}^{\gamma_{j}^{-1} \gamma_{i}}(\gamma)\right)>\alpha\right\}\right)<n^{-2}
$$

for all $1 \leq i<j \leq n$. Translating by $\gamma_{j}$,

$$
\mu\left(\left\{\gamma \in \Gamma \mid \forall h \leq k, v\left(f_{h}^{\gamma_{j}}(\gamma)\right)>\alpha, v\left(f_{h}^{\gamma_{i}}(\gamma)\right)>\alpha\right\}\right)<n^{-2} .
$$

Let

$$
S_{j}=\left\{\gamma \in \Gamma \mid \forall h \leq k, v\left(f_{h}^{\gamma_{j}}(\gamma)\right)>\alpha\right\} .
$$

By inclusion-exclusion,

$$
1-\sum_{1 \leq i \leq n} \mu\left(S_{i}\right)+\sum_{1 \leq i<j \leq n} \mu\left(S_{i} \cap S_{j}\right) \geq 0 .
$$

As

$$
\mu\left(S_{j}\right)=\mu\left(\left\{\gamma \in \Gamma \mid \forall h \leq k, v\left(f_{h}(\gamma)\right)>\alpha\right\}\right)
$$

for all $j,(1)$ implies

$$
\mu\left(\left\{\gamma \in \Gamma \mid \forall h \leq k, v\left(f_{h}(\gamma)\right)>\alpha\right\}\right) \leq \frac{1+\left(\begin{array}{l}
n \\
2
\end{array}\right) n^{-2}}{n}<\frac{2}{n},
$$


where $n$ can be taken to be as large as we wish. The induction is complete and the proposition follows.

As a more or less immediate consequence (of the proof) of Proposition 2.3 and the classical Cebotarev density theorem we have:

Theorem 2.4. Let $F$ be a global field, $F^{s}$ a separable closure, and $G_{F}=$ $\operatorname{Gal}\left(F^{s} / F\right)$. Let $V$ be a valuation ring with residue characteristic $\ell$ and characteristic-zero fraction field $K$. Let $\rho: G_{F} \rightarrow \mathrm{GL}_{n}(K)$ be a continuous, finitely ramified representation. Let $X$ be a nowhere dense closed subset of the Zariski closure of $\rho\left(G_{F}\right)$ which is invariant under conjugation by $\rho\left(G_{F}\right)$. Then the set of places of $F$ at which the Frobenii under $\rho$ lie in $X$ has Dirichlet density 0 .

Proof. The referee has pointed out that we need to comment on the link between Dirichlet density and Haar measures which allows us to deduce the theorem from Proposition 2.3. We owe the following comments to the referee. Since $X$ is a closed set and $\rho$ is continuous, the preimage of $X$ in the quotient of $G_{F}$ through which $\rho$ factors is a closed set. Furthermore, by (the proof of) Proposition 2.3 the preimage of $X$ has Haar measure zero as $X$ is assumed to be nowhere dense. In particular, its boundary has Haar measure zero. Hence, by Corollary 2 in Section 2.2 of [S1] we infer that the natural density of the set of places with Frobenius in $X$ exists and is zero. Thus, the Dirichlet density also exists and is zero.

In the case when $\rho$ is finitely ramified, we do not know if quantitative refinements of the density 0 result, analogous to Théorème 10 of [S2], are true in this general setting. In any case our "soft" techniques will not yield such quantitative refinements. Theorem 2.5 of the next section allows to us to remove the hypothesis of finite ramification in Theorem 2.4. However with the removal of this hypothesis quantitative refinements cannot be expected (as the last section of $[\mathrm{KLR}]$ shows).

2.2. Ramification. Here is the main theorem of this section.

Theorem 2.5. Let $F$ be a global field, $F^{s}$ an algebraic closure, and $G_{F}=$ $\operatorname{Gal}\left(F^{s} / F\right)$. Let $V$ be a valuation ring with residue characteristic $\ell$ unequal to the characteristic of $F$. Furthermore suppose $V$ has characteristic-zero fraction field $K$. Let $\rho: G_{F} \rightarrow \mathrm{GL}_{n}(K)$ be a continuous semisimple representation. Then the set of places of $F$ at which $\rho$ is ramified has Dirichlet density 0.

To prove the main theorem, we need two simple lemmas.

Lemma 2.6. Let $q>1$ be a positive integer, $L$ an algebraically closed field, and $x$ and $y$ elements of $\mathrm{GL}_{n}(L)$ such that $x y x^{-1}=y^{q}$. Then either $y$ is semisimple and of finite order in $\mathrm{GL}_{n}(L)$ or there exist eigenvalues $\lambda_{1}$ and $\lambda_{2}$ of $x$ with $\lambda_{1} / \lambda_{2}=q$. 
Proof. Let $y=y_{s} y_{u}$ be the multiplicative Jordan decomposition. Then

$$
x y_{s} x^{-1}=y_{s}^{q}, x y_{u} x^{-1}=y_{u}^{q} .
$$

If the characteristic polynomial of $y_{s}$ is $\prod_{i=1}^{n}\left(x-\lambda_{i}\right)$, then there exists a permutation $\pi \in S_{n}$ such that

$$
\lambda_{i}^{q}=\lambda_{\pi(i)}
$$

which implies that $\lambda_{i}^{\left(q^{j_{i}}-1\right)}=1$ for some $j_{i} \leq n$. Thus

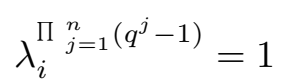

so either $y$ is semisimple of finite order or $y_{u} \neq 1$.

In the latter case, using the relation that $x$ and $y_{u}$ satisfy, it is easy to see that $x$ and $y_{u}$ are in $B(L)$ where $B$ is the Borel subgroup stabilising some maximal flag of $L^{n}$ (see the discussion in Section 3 of [KLR] three paragraphs before the statement of Theorem 19). Let

$$
U_{1}=[B, B], U_{2}=\left[B, U_{1}\right], \ldots, U_{n}=\{1\}
$$

denote the descending central series. Thus $y_{u} \in U_{1}$ but $y_{u} \notin U_{n}$. Choose $k$ so that $y_{u} \in U_{k} \backslash U_{k+1}$. Then via the action of $B(L) / U_{1}(L)$ on $U_{k}(L) / U_{k+1}(L)$ we see that $\bar{y}_{u}$ is an eigenvector of $\bar{x} \in B(L) / U_{1}(L)$ with eigenvalue $q$. As the eigenvalues of the diagonal matrix $\sum \lambda_{i} e_{i i}$ acting on $U_{k}(L) / U_{k+1}(L)$ are

$$
\lambda_{i} \lambda_{i+k}^{-1}, 1 \leq i \leq n-k
$$

the lemma follows.

Lemma 2.7. In every valuation ring $V, 1$ has an open neighborhood in which the only root of unity is 1 itself.

Proof. For $\alpha>0$ in the value group of $V, v(x-1) \geq \alpha$ implies $v\left(x^{n}-1\right) \geq \alpha$. It therefore suffices to find $\alpha$ such that for every prime $p$, and every primitive $p$ th root of unity $\zeta_{p} \in V, v\left(\zeta_{p}-1\right) \leq \alpha$. As $\zeta_{p}-1$ divides $p, v\left(\zeta_{p}-1\right) \leq v(p)$. There is at most one non-zero rational prime $p$ in $\mathbb{Z}$ for which $v(p)>0$, so we can take $\alpha:=\sup _{p \neq 0} v(p)<\infty$.

Proof of Theorem 2.5. Choose an integral model of $\rho$ by Lemma 2.2. The representation $\rho$ can be wildly ramified at $\wp$ only if $\wp$ has the same residue characteristic as $V$ or the residual representation that arises from reducing the chosen integral model of $\rho$ is ramified at $\wp$. Henceforth we will ignore this finite set of primes. If $\wp$ is a prime of $F$ and $G_{\wp}$ and $T_{\wp}$ denote the Galois group of the maximal tamely ramified extension of the completion $F_{\wp}$ and the tame inertia subgroup respectively, we have a short exact sequence

$$
0 \rightarrow T_{\wp} \rightarrow G_{\wp} \rightarrow \hat{\mathbb{Z}} \rightarrow 0 .
$$


The quotient group $\hat{\mathbb{Z}}$ is topologically generated by the Frobenius class $\sigma_{\wp}, T_{\wp}$ is topologically generated by some non-canonical class $\tau_{\wp}$, and

$$
\sigma_{\wp} \tau_{\wp} \sigma_{\wp}^{-1}=\tau_{\wp}^{\|\wp\|} \text {. }
$$

By Lemma 2.7, there exists an open neighborhood $G_{F^{\prime}}$ of the identity in $G_{F}$ such that no element of $\rho\left(G_{F^{\prime}}\right)$ can have an eigenvalue which is a non-trivial root of unity. We exclude henceforth from discussion the finite set of primes $\wp^{\prime}$ in the finite extension $F^{\prime}$ which are ramified over $F$. Thus the natural maps $G_{\wp^{\prime}} \rightarrow G_{\wp}$ restrict to isomorphisms $T_{\wp^{\prime}} \rightarrow T_{\wp}$. It follows using Lemma 2.6 that $\rho\left(\tau_{\wp}\right)$ is unipotent for all primes $\wp$ that we consider. In particular, $\rho\left(\tau_{\wp}\right)$ has infinite order if it is non-trivial, as $K$ is characteristic-zero.

The semisimplicity of $\rho$ implies $G^{\circ}$ is a reductive group. Let $G$ be the Zariskiclosure of $\rho\left(G_{F}\right)$ and $Z$ the center of the identity component $G^{\circ}$. Choose a faithful $K$-representation $G / Z \hookrightarrow \mathrm{GL}_{m}$. As 1 is the only unipotent element in $Z(K), T_{\wp}$ lies in the kernel of the composition map

$$
G_{F} \rightarrow G(K) \rightarrow(G / Z)(K) \rightarrow \mathrm{GL}_{m}(K)
$$

if and only if $\rho$ is unramified at $\wp$. Replacing $\rho$ by this composition if necessary, we assume without loss of generality that $G$ has semisimple identity component.

By (2) and Lemma 2.6, either $\rho$ is unramified at $\wp$ or $\rho\left(\sigma_{\wp}\right)$ has two eigenvalues in $\bar{K}$ whose ratio is $\|\wp\|$. Let $\varepsilon$ denote the $\ell$-adic cyclotomic character. Consider the direct sum

$$
\alpha=\rho \oplus \varepsilon: G_{F} \rightarrow G(K) \times \mathrm{GL}_{1}(K),
$$

and let $H$ denote the Zariski closure of $\alpha\left(G_{F}\right)$ in $G \times \mathrm{GL}_{1}$. Thus $H \subset G \times$ $\mathrm{GL}_{1}$ and $H$ projects onto each factor. We claim $H=G \times \mathrm{GL}_{1}$ by "Goursat's lemma for algebraic groups in characteristic zero." Explicitly, let $H_{1}$ and $H_{2}$ denote the scheme-theoretic kernels of the projection maps $H \rightarrow G$ and $H \rightarrow$ $\mathrm{GL}_{1}$ respectively; as the characteristic of $K$ is zero, these are algebraic groups and moreover normal subgroups of $\mathrm{GL}_{1}$ and $G$ respectively. Now $H /\left(H_{1} H_{2}\right)$ is contained in $\left(G / H_{2}\right) \times\left(\mathrm{GL}_{1} / H_{1}\right)$ and maps isomorphically to each factor. Therefore, $G / H_{2}$ is isomorphic to $\mathrm{GL}_{1} / H_{1}$. Every quotient of $\mathrm{GL}_{1}$ is a torus and $G$ admits no non-trivial toric quotient, so $H_{1}=\mathrm{GL}_{1}, H_{2}=G$, and $H=$ $H_{1} H_{2}=G \times \mathrm{GL}_{1}$.

Let $X \subset H$ denote the subset of pairs $(g, c) \in H$ such that $g$ and $g c$ have at least one eigenvalue in common. This is a closed subscheme, being the zero locus of the resultant of the characteristic polynomials of $g$ and $g c$ (as points of $\mathrm{GL}_{m}$ ). For each $g$ there are only finitely many possible values of $c$ such that $(g, c) \in X$, so $X$ is of codimension $\geq 1$ in each component of $H$. By the construction of $X$, if $\alpha$ is ramified at $\wp$, then for any choice of $\sigma_{\wp}, \alpha\left(\sigma_{\wp}\right)$ lies in $X$. By Proposition 2.3 (with $\mu$ Haar measure on $\alpha\left(G_{F}\right)$ ), for any $\epsilon>0$, we can find an open and closed neighborhood $N_{\epsilon}$ of $X(K)$ in $H(K)$ such that $\mu\left(\alpha\left(G_{F}\right) \cap N_{\epsilon}\right)<\epsilon$. Let $F^{\prime \prime}$ be a finite Galois extension of $F$ such that $\alpha^{-1}\left(N_{\epsilon}\right)$ is a finite union of $G_{F^{\prime \prime}}$ cosets. Thus the image of $\alpha^{-1}(X(K))$ in $\operatorname{Gal}\left(F^{\prime \prime} / F\right)$ has less than $\epsilon\left|\operatorname{Gal}\left(F^{\prime \prime} / F\right)\right|$ elements and is a union of conjugacy classes in $\operatorname{Gal}\left(F^{\prime \prime} / F\right)$ (since $X$ is stable 
under conjugation in $H$ ). By the Cebotarev density theorem, there is a set of primes $\wp$ of $F$ of Dirichlet density at least $1-\epsilon$ such that $\rho$ is unramified at $\wp$. The theorem follows.

Remark: As almost all ramification is unipotent, the proof also shows that if $\rho$ has abelian image it is finitely ramified.

\section{Fields of definition of finitely ramified representations}

In Theorem 4.1 of the next section we construct a continuous transcendental representation $\rho: G_{\mathbb{Q}} \rightarrow \mathrm{GL}_{2}\left(\mathbb{C}_{\ell}\right)$,

- which is semisimple, and therefore unramified at a density one set of places by Theorem 2.5 ,

- at a density one set of unramified places has characteristic polynomials of Frobenii defined over $\overline{\mathbb{Q}}_{\ell}$,

- does not have a model over $\overline{\mathbb{Q}}_{\ell}$, or equivalently over any finite extension of $\mathbb{Q}_{\ell}$.

In this section we show that examples such as in Theorem 4.1 are necessarily infinitely ramified (although in the example of the next section we could simply ensure this during the construction).

Theorem 3.1. Let $G$ be a topologically finitely generated profinite group, and $\rho: G \rightarrow \mathrm{GL}_{n}\left(\mathbb{C}_{\ell}\right)$ a continuous homomorphism. Let $\left\{x_{i}\right\}$ be a set of elements of $G$ such that the union of the conjugacy classes of the $x_{i}$ 's is dense in $G$. Assume $\operatorname{tr}\left(\rho\left(x_{i}\right)\right) \in \overline{\mathbb{Q}}_{\ell}$ for all $i$. Then there exists a finite extension $L$ of $\mathbb{Q}_{\ell}$ such that $\operatorname{tr}(\rho(g)) \in L$ for all $g \in G$. If $\rho$ is semisimple, replacing $L$ by a finite extension if necessary, $\rho(G)$ can be conjugated into a subgroup of $\mathrm{GL}_{n}(L)$.

Before proving the theorem we derive a corollary that is of particular relevance to this paper.

Corollary 3.2. Let $F$ be a global field with Galois group $G_{F}=\operatorname{Gal}\left(F^{s} / F\right)$, $\ell$ a rational prime different from the characteristic of $F$, and $\rho: G_{F} \rightarrow \mathrm{GL}_{n}\left(\mathbb{C}_{\ell}\right)$ a continuous, irreducible Galois representation ramified at a finite set of places. If there exists a set of places $\wp$ of $F$ of Dirichlet density 1 such that the trace of Frobenius $\operatorname{tr}\left(\rho\left(\sigma_{\wp}\right)\right)$ belongs to $\overline{\mathbb{Q}}_{\ell} \subset \mathbb{C}_{\ell}$, then $\rho$ is conjugate to a representation with values in a finite extension of $\mathbb{Q}_{\ell}$.

Proof. Let $F^{\prime}$ denote the field such that $\rho$ factors through $\operatorname{Gal}\left(F^{\prime} / F\right)$. Only finitely many places of $F$ ramify in $F^{\prime}$ by assumption. We first note that the Cebotarev density theorem implies that for any set of places $\{\wp\}$ of $F$, unramified in $F^{\prime}$, of Dirichlet density 1, the union of the Frobenius conjugacy classes $\sigma_{\wp}$ is dense in $\operatorname{Gal}\left(F^{\prime} / F\right)$. Now the corollary follows from Theorem 3.1 (and Lemma 2.2) using the following consequence of the Hermite-Minkowski theorem: the Galois group of a pro- $\ell$ extension of a global field $L$ (with $\ell$ different from the characteristic of $L$ ), ramified only over a finite set of primes $S$, is topologically finitely generated. 
Now we return to the proof of the theorem.

Proof of Theorem 3.1: By Lemma 2.2, $\rho$ has a model over the valuation ring $\mathcal{O}$ of $\mathbb{C}_{\ell}$, and thus we consider $\rho$ as taking values in $\mathrm{GL}_{n}(\mathcal{O})$. By continuity of $\rho$ we see $\rho \bmod \ell^{m}$ has finite image for all $m$. Thus $\rho \bmod \ell^{m}$ can be regarded as taking values in $\mathrm{GL}_{n}\left(\mathcal{O}_{L_{m}} / \ell^{m} \mathcal{O}_{L_{m}}\right)$ where $\mathcal{O}_{L_{m}}$ is the ring of integers of a finite extension $L_{m}$ of $\mathbb{Q}_{\ell}$. This can be all be arranged because of the surjectivity of the map $\overline{\mathbb{Z}}_{\ell} \rightarrow \mathcal{O} / \ell^{m} \mathcal{O}$. The residual homomorphism that arises from reducing $\rho$ modulo the maximal ideal of $\mathcal{O}$ is a homomorphism $\bar{\rho}: G_{F} \rightarrow \mathrm{GL}_{n}(k)$ for a $k / \mathbb{F}_{\ell}$ a finite extension of $\mathbb{F}_{\ell}$ that we may take to be the residue field of $L_{1}$. We assume for convenience that $L_{i}$ are Galois over $\mathbb{Q}_{\ell}$, and also that $L_{m} \subset L_{m+1}$.

Consider the category $\mathcal{C}$ whose objects are complete local Noetherian $W(k)$ algebras with residue field $k$ and whose morphisms are local morphisms of $W(k)$ algebras that induce the identity map on residue fields. For $A$ in $\mathcal{C}$, consider deformations of $\bar{\rho}$ to $\mathrm{GL}_{n}(A)$, i.e., continuous homomorphisms $G \rightarrow \mathrm{GL}_{n}(A)$ that reduce to $\bar{\rho}$ modulo the maximal ideal of $A$, taken up to conjugation by matrices that reduce to the identity. By the standard theory (see section 20 of $[\mathrm{M}]$ ), using that $G$ is finitely generated topologically, there is a versal deformation $\rho_{\mathcal{R}}: G_{F} \rightarrow \mathrm{GL}_{n}(\mathcal{R})$ with $\mathcal{R} \in \mathcal{C}$.

We need a lemma:

Lemma 3.3. There is continuous map $\pi: \mathcal{R} \rightarrow \mathcal{O}$ of local rings such that the representation $\pi_{\circ} \rho_{\mathcal{R}}$ has the same traces as $\rho$.

Proof. Consider $\rho \bmod \ell^{s}$ for any $s>0$. This is valued in $\mathrm{GL}_{n}\left(\mathcal{O}_{L_{s}} / \ell^{s} \mathcal{O}_{L_{s}}\right)$ by what was said above. For each positive integer $s$ consider $A_{s}=\left(\mathcal{O}_{L_{1}}+\ell \mathcal{O}_{L_{2}}+\right.$ $\left.\cdots+\ell^{s-1} \mathcal{O}_{L_{s}}\right) / \ell^{s} \mathcal{O}_{L_{s}}$. Then it is easy to see that $A_{s}$ is an object of $\mathcal{C}$ and $\rho$ $\bmod \ell^{s}$ is valued in $\operatorname{GL}_{n}\left(A_{s}\right)$, via the natural inclusion $A_{s} \hookrightarrow \mathcal{O} / \ell^{s} \mathcal{O}$. It is also easy to see by inspection that if we reduce $A_{s}$ modulo its ideal $\ell^{s-1} \mathcal{O}_{L_{s}}$ we get $A_{s-1}$. By the versal property of $\mathcal{R}$, we have a morphism $\pi_{s}: \mathcal{R} \rightarrow A_{s}$ such that $\pi_{s} \circ \rho_{\mathcal{R}}$ is isomorphic to (the chosen integral model of) $\rho \bmod \ell^{s}$. Here we are using the fact that the image of $\rho \bmod \ell^{s}$ is valued in the ring that is the image of the composition of inclusions $A_{s} \hookrightarrow \mathcal{O}_{L_{s}} / \ell^{s} \mathcal{O}_{L_{s}} \hookrightarrow \mathcal{O} / \ell^{s} \mathcal{O}$ for all $s>0$. In particular the traces of $\pi_{s} \circ \rho_{\mathcal{R}}$ and $\rho \bmod \ell^{s}$ coincide. We observe that as the rings $A_{s}$ have finite cardinality and as $\mathcal{R}$ is topologically finitely generated, for each $s$ there are only finitely many morphisms $\alpha_{s}: \mathcal{R} \rightarrow A_{s}$. In particular, there are only finitely many $\alpha_{s}$ with the further property that $\alpha_{s} \circ \rho_{\mathcal{R}}$ is isomorpic to $\rho \bmod \ell^{s}$. By a standard compactness argument, there exists a compatible subsequence $\alpha_{t_{1}}, \alpha_{t_{2}}, \ldots$ of homomorphisms, which gives a homomorphism $\pi: \mathcal{R} \rightarrow \mathcal{O}$ such that $\pi \circ \rho_{\mathcal{R}}$ has the same traces as $\rho$. In fact the $\pi$ we have constructed even has the property that $\pi \circ \rho_{\mathcal{R}}$ is isomorphic to the chosen integral model of $\rho$. 
The image $\mathcal{S}$ of $\mathcal{R}$ in $\mathcal{O}$ is a quotient of a complete Noetherian $W(k)$-algebra and is therefore a complete Noetherian $W(k)$-algebra itself. We have the following key lemma whose proof we owe to Shankar Sen:

Lemma 3.4. Let $\mathcal{S}$ be as above. Let $K$ denote the field of fractions of $W(k)$. Then there exists a finite subextension $L$ of $K \subset \mathbb{C}_{\ell}$ such that the integral closure of $W(k)$ in $\mathcal{S} \subset \mathbb{C}_{\ell}$ is contained in $L$.

Proof. As $\mathcal{S} \subset \mathbb{C}_{\ell}$, it is a complete Noetherian integral domain, so by Cohen structure theory ([EGA IV, $\left.0_{\text {IV }} 19.8 .8\right]$ ), there exists a subring $\mathcal{S}_{0} \subset \mathcal{S}$ such that $\mathcal{S}_{0} \cong W(k)\left[\left[u_{1}, \ldots, u_{r}\right]\right]$, and $\mathcal{S}$ is a local, module-finite $\mathcal{S}_{0}$-algebra. Suppose it can be generated by $s$ elements. If $\mathcal{T}$ denotes the integral closure of $W(k)$ in $\mathcal{S}$, every element $t \in \mathcal{T}$ is integral over $\mathcal{S}_{0}$ and therefore satisfies a minimal monic polynomial equation with coefficients in $\mathcal{S}_{0}$ and degree $\leq s$. This polynomial divides the minimal polynomial of $t$ over $W(k)$ and therefore has coefficients in $\overline{\mathbb{Q}}_{\ell} \cap W(k)\left[\left[u_{1}, \ldots, u_{r}\right]\right]=W(k)$. It follows that $t$ lies in an extension of $K$ of degree $\leq s$. By Krasner's lemma, the compositum of all such extensions is a finite extension of $K$.

We return to the proof of Theorem 3.1. Let $X$ denote the union of the conjugacy classes of the $x_{i} \in G$. We know that $\operatorname{tr}(\rho)$ is continuous and maps $X$ into $L$, $X$ is dense in $G$, and $L$ is closed in $\mathbb{C}_{\ell}$. It follows that $\operatorname{tr}(\rho)$ maps $G$ into $L$. If $\rho$ is semisimple, the theory of pseudorepresentations for instance (see Theorem 1 of $[\mathrm{T}])$ shows that we can conjugate $\rho(G)$ into $\mathrm{GL}_{n}\left(\overline{\mathbb{Q}}_{\ell}\right)$, and thence into $\mathrm{GL}_{n}\left(L^{\prime}\right)$ for $L^{\prime} / L$ a finite extension by the usual Baire category argument of the introduction.

Remark: Mark Dickinson has remarked that one does not need the formalism of deformation theory in the proof of Theorem 3.1. But it seems to be worth checking as in proof of Lemma 3.3 that deformations to $\mathbb{C}_{\ell}$ also arise as specialisations of the versal deformation.

\section{An example}

We assume $\ell \geq 5$ in this section. Frobenius at a prime $r$ is denoted $\sigma_{r}$. The main result of this section is:

Theorem 4.1. There exists a potentially semistable 2-dimensional continuous irreducible Galois representation $\rho: G_{\mathbb{Q}}:=\operatorname{Gal}(\overline{\mathbb{Q}} / \mathbb{Q}) \rightarrow \mathrm{GL}_{2}\left(\mathbb{C}_{\ell}\right)$ (for each prime $\ell \geq 5)$, such that for primes $r$ in a set of density one the trace $\operatorname{tr}\left(\sigma_{r}\right)$ of Frobenius at $r$ lies in $\overline{\mathbb{Q}}_{\ell} \subseteq \mathbb{C}_{\ell}$. Furthermore $\rho$ is not conjugate to any representation with values in $\overline{\mathbb{Q}}_{\ell}$.

We now develop the machinery needed to prove Theorem 4.1.

Lemma 4.2. Let $\ell \geq 5$ and $B_{m} \subset \mathrm{GL}_{2}\left(W\left(\mathbb{F}_{\ell^{d}}\right) / \ell^{m} W\left(\mathbb{F}_{\ell^{d}}\right)\right)$ be a subgroup whose mod $\ell$ reduction is $\mathrm{GL}_{2}\left(\mathbb{F}_{\ell}\right)$. Then, up to conjugation by an element of 
the form $I+\ell X \in \mathrm{GL}_{2}\left(W\left(\mathbb{F}_{\ell^{d}}\right) / \ell^{m} W\left(\mathbb{F}_{\ell^{d}}\right)\right), B_{m}$ contains an element $\left(\begin{array}{cc}a & 0 \\ 0 & 1\end{array}\right)$ where $a \in\left(\mathbb{Z} / \ell^{m} \mathbb{Z}\right)^{\times}$and $a \not \equiv \pm 1 \bmod \ell$.

Proof. By hypothesis we know that the $\bmod \ell$ reduction of $B_{m}$ contains an element $h$ of the form $\left(\begin{array}{ll}a & 0 \\ 0 & 1\end{array}\right)$ where $a \in(\mathbb{Z} / \ell \mathbb{Z})^{\times}$and $a \not \equiv \pm 1 \bmod \ell$. Choose any lift $g \in B_{m}$ of this mod $\ell$ element $h$. As the characteristic polynomial of $h$ has distinct roots in $\mathbb{F}_{\ell}$, and as $\ell>2$, the element $g$ can be conjugated to a diagonal matrix viewed as an element of $\mathrm{GL}_{2}\left(W\left(\mathbb{F}_{\ell^{d}}\right) / \ell^{m} W\left(\mathbb{F}_{\ell^{d}}\right)\right)$. By taking $\ell^{r}$ th powers, for large enough $r$, we obtain an element that has a conjugate in $B_{m}$ with the desired properties.

Remark: We need Lemma 4.2 as an ingredient for Fact 4.6. In [KLR] our $\rho_{m}$ always had image $\mathrm{GL}_{2}\left(\mathbb{Z} / \ell^{m} \mathbb{Z}\right)$ so it obviously contained elements like those in Lemma 4.2.

Definition 4.3. We say a Galois representation $\bar{\rho}$ satisfies our running hypotheses if

- $\bar{\rho}: \operatorname{Gal}(\overline{\mathbb{Q}} / \mathbb{Q}) \rightarrow \mathrm{GL}_{2}(\mathbb{Z} / \ell \mathbb{Z})$ is surjective with $\ell \geq 5$,

- $\operatorname{det}(\bar{\rho})=\varepsilon$, the cyclotomic character.

In this section we assume, primarily for simplicity, that all determinants are the cyclotomic character $\varepsilon$. We denote by $\operatorname{Ad}^{0} \bar{\rho}$ the Galois module of $2 \times 2$ trace zero matrices over $\mathbb{F}_{\ell}$ with Galois action through $\bar{\rho}$ via conjugation. We denote by $\left(\operatorname{Ad}^{0} \bar{\rho}\right)^{*}$ the Cartier dual of $\operatorname{Ad}^{0} \bar{\rho}$. Let $S$ be a finite set of primes that contains the primes at which $\bar{\rho}$ is ramified. Let $J=\mathbb{Q}\left(\operatorname{Ad}^{0} \bar{\rho}, \mu_{\ell}\right)$. For $\phi \in$ $H^{1}\left(\operatorname{Gal}(\overline{\mathbb{Q}} / \mathbb{Q}),\left(\operatorname{Ad}^{0} \bar{\rho}\right)^{*}\right)$ the symbol $J_{\phi}$ denotes the field fixed by the kernel of $\left.\phi\right|_{G_{J}}$. By Lemma 9 of $[\mathrm{R} 1]$ we see that, as an $\mathbb{F}_{\ell}[\operatorname{Gal}(\mathrm{J} / \mathbb{Q})]$-module, $\operatorname{Gal}\left(\mathrm{J}_{\phi} / \mathrm{J}\right)$ is isomorphic to $\left(\operatorname{Ad}^{0} \bar{\rho}\right)^{*}$. Finally, the kernel of the localisation map $H^{i}\left(G_{X}, M\right) \rightarrow$ $\Pi_{v \in X} H^{i}\left(G_{v}, M\right)$ is denoted $\amalg_{X}^{i}(M)$.

We recall some Definitions, Facts, Lemmas and Propositions from [KLR]. The precise $[\mathrm{KLR}]$ references are given parenthetically in each case.

Definition 4.4. (A slight variant of Definition 1 of $[K L R]$ ) Suppose $\bar{\rho}$ satisfies our running hypotheses. We say a prime $q$ is nice (for $\bar{\rho}$ ) if

- $q \neq \ell$ and $q$ is $n o t \pm 1 \bmod \ell$,

- $\bar{\rho}$ is unramified at $q$,

- the eigenvalues of $\bar{\rho}\left(\sigma_{q}\right)$ (where $\sigma_{q}$ is Frobenius at $q$ ) have ratio $q$.

Let $\rho_{m}$ be a deformation of $\bar{\rho}$ to $\mathrm{GL}_{2}\left(W\left(\mathbb{F}_{\ell^{d}}\right) / \ell^{m} W\left(\mathbb{F}_{\ell^{d}}\right)\right)$ with determinant the cyclotomic character. We say a prime $q$ is $\rho_{m}$-nice if

- $q$ is nice for $\bar{\rho}$,

- $\rho_{m}$ is unramified at $q$, and the (necessarily distinct) roots of the characteristic polynomial of $\rho_{m}\left(\sigma_{q}\right)$ have ratio $q$. Note that since $q$ is nice, the $\bmod \ell^{m}$ characteristic polynomial of $\rho_{m}\left(\sigma_{q}\right)$ has distinct roots that are units; it follows that the eigenvalues of $\rho_{m}\left(\sigma_{q}\right)$ are well-defined in $W\left(\mathbb{F}_{\ell^{d}}\right) / \ell^{m} W\left(\mathbb{F}_{\ell^{d}}\right)$. 
Fact 4.5. (Lemma 6 of $[\mathrm{KLR}]$ ) Let $\bar{\rho}$ satisfy our running hypotheses. There exists a finite set $T$ of nice primes such that $\amalg_{S \cup T}^{1}\left(\operatorname{Ad}^{0} \bar{\rho}\right)$ and $\amalg_{S \cup T}^{2}\left(\operatorname{Ad}^{0} \bar{\rho}\right)$ are trivial. After enlarging $S$, we may thus assume $\amalg_{S}^{1}\left(\operatorname{Ad}^{0} \bar{\rho}\right)$ and $\amalg_{S}^{2}\left(\operatorname{Ad}^{0} \bar{\rho}\right)$ are trivial.

Fact 4.6. (Weak variant of Fact 5 of $[\mathrm{KLR}]$ ) Let $\bar{\rho}$ satisfy our running hypotheses. Let $\rho_{m}$ be a deformation of $\bar{\rho}$ to $\mathrm{GL}_{2}\left(W\left(\mathbb{F}_{\ell^{d}}\right) / \ell^{m} W\left(\mathbb{F}_{\ell^{d}}\right)\right)$ unramified outside $S$ with $\operatorname{det}\left(\rho_{m}\right)=\varepsilon$. Suppose $\left\{\phi_{1}, \ldots, \phi_{r}\right\}$ is independent in

$H^{1}\left(\operatorname{Gal}(\overline{\mathbb{Q}} / \mathbb{Q}),\left(\operatorname{Ad}^{0} \bar{\rho}\right)^{*}\right)$. Let $J$ be a subset of $\{1, \ldots, r\}$. Then there exists a Cebotarev set $X$ (a set $X$ of primes of positive density coming from an application of Cebotarev's Theorem) of primes $w \notin S$ such that

- $w$ is $\rho_{m}$-nice,

- $\left.\phi_{j}\right|_{G_{w}} \neq 0$ for $j \in J$ and $\left.\phi_{j}\right|_{G_{w}}=0$ for $j \in\{1, \ldots, r\} \backslash J$.

Proof. Lemma 4.2 implies the image of $\rho_{m}$ contains (up to conjugation) an element $\left(\begin{array}{ll}a & 0 \\ 0 & 1\end{array}\right)$ with $a \in\left(\mathbb{Z} / \ell^{m} \mathbb{Z}\right)^{*}$ and $a \not \equiv \pm 1 \bmod \ell$. Using the fact that $\operatorname{det}\left(\rho_{m}\right)=\varepsilon$, one sees from the proof of Fact 5 of [KLR] that the Cebotarev set of primes with Frobenius in the conjugacy class of this element and the desired splitting properties in the fields $J_{\phi_{i}}$ provides the $\rho_{m}$-nice primes.

Remarks: 1) It is not true in Fact 4.6 that for $m>1$ one can also include splitting properties for independent elements of $H^{1}\left(\operatorname{Gal}(\overline{\mathbb{Q}} / \mathbb{Q}), \operatorname{Ad}^{0} \bar{\rho}\right)$. One can obtain a result including splitting properties for independent elements of both $H^{1}\left(\operatorname{Gal}(\overline{\mathbb{Q}} / \mathbb{Q}), \operatorname{Ad}^{0} \bar{\rho}\right)$ and $H^{1}\left(\operatorname{Gal}(\overline{\mathbb{Q}} / \mathbb{Q}),\left(\operatorname{Ad}^{0} \bar{\rho}\right)^{*}\right)$ for $\mathrm{GL}_{2}\left(W\left(\mathbb{F}_{\ell^{d}}\right) / \ell^{m} W\left(\mathbb{F}_{\ell^{d}}\right)\right)$ representations provided the minimal field of definition of the adjoint representation is $\mathbb{F}_{\ell^{d}}$. (See Lemma 9 of [R3] for the case $d=1$ ). In our situation the minimal field of definition of the adjoint representation is always $\mathbb{F}_{\ell}$.

2) While Fact 4.5 above requires the full strength of Fact 5 of [KLR], Lemma 4.7 and Proposition 4.8 here only require the weaker Fact 4.6 above.

Lemma 4.7. (Lemma 8 of $[\mathrm{KLR}])$ Let $\bar{\rho}$ satisfy our running hypotheses. Let $\rho_{m}$ be a deformation of $\bar{\rho}$ to $\mathrm{GL}_{2}\left(W\left(\mathbb{F}_{\ell^{d}}\right) / \ell^{m} W\left(\mathbb{F}_{\ell^{d}}\right)\right)$ unramified outside a set S. Suppose $\amalg_{S}^{1}\left(\operatorname{Ad}^{0} \bar{\rho}\right)$ and $\amalg_{S}^{2}\left(\operatorname{Ad}^{0} \bar{\rho}\right)$ are trivial and $\operatorname{det}\left(\rho_{m}\right)=\varepsilon$. Let $R$ be any finite collection of unramified primes of $\rho_{m}$ disjoint from $S$.

There is a finite set $Q=\left\{q_{1}, \ldots, q_{n}\right\}$ of $\rho_{m}$-nice primes disjoint from $R \cup S$ such that the map

$$
H^{1}\left(G_{S \cup Q}, \operatorname{Ad}^{0} \bar{\rho}\right) \rightarrow\left(\oplus_{\mathrm{v} \in \mathrm{S}} \mathrm{H}^{1}\left(\mathrm{G}_{\mathrm{v}}, \operatorname{Ad}^{0} \bar{\rho}\right)\right) \oplus\left(\oplus_{\mathrm{r} \in \mathrm{R}} \mathrm{H}_{\mathrm{nr}}^{1}\left(\mathrm{G}_{\mathrm{r}}, \operatorname{Ad}^{0} \bar{\rho}\right)\right)
$$

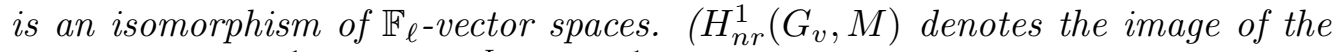
inflation map $\left.H^{1}\left(G_{v} / I_{v}, M^{I_{v}}\right) \rightarrow H^{1}\left(G_{v}, M\right)\right)$. Upon tensoring with $\mathbb{F}_{\ell^{d}}$ they can be viewed as isomorphisms of $\mathbb{F}_{\ell^{d}}$ vector spaces.

Proof. The only difference between here and situation in [KLR] is that the deformation is to the Witt ring. Fact 4.6 provides us with the necessary $\rho_{m}$-nice primes for the proof of the Proposition. 
Proposition 4.8. (Proposition 10 of $[\mathrm{KLR}]$ ) Let the notations be as in Lemma 4.7. For each $k=1, \ldots, n$ there is a set $\tilde{T}_{k}$ of one or two primes of $T_{k}$ and an element $f_{k} \in H^{1}\left(G_{S \cup Q \cup \tilde{T}_{k}}, \operatorname{Ad}^{0} \bar{\rho}\right)$ such that

- $f_{k}\left(\sigma_{t_{k}}\right)=0$ for all $t_{k} \in \tilde{T}_{k}$ and $\left.f_{k}\right|_{G_{q_{k}}} \neq 0$,

- $j<k$ implies that for $t_{j} \in \tilde{T}_{j}$ we have $f_{k}\left(\sigma_{t_{j}}\right)=0$,

- $\left.f_{k}\right|_{G_{v}}=0$ for all $v \in S \cup R \cup Q \backslash\left\{q_{k}\right\}$.

Proof. The proof from $[\mathrm{KLR}]$ carries over word for word.

Sketch of the proof of Theorem 4.1 The proof follows the general strategy of the proof of Application I of [KLR].

- Let $\rho_{1}=\bar{\rho}$ satisfy our running hypotheses. One can for instance take $\rho_{1}$ to be the Galois action on the $\ell$-division points of an non-CM elliptic curve over $\mathbb{Q}$ for $\ell$ large enough.

- Use Fact 4.5 to enlarge $S$ to $S_{1}$ for which $\amalg_{S_{1}}^{1}\left(\operatorname{Ad}^{0} \bar{\rho}\right)$ and $\amalg_{S_{1}}^{2}\left(\operatorname{Ad}^{0} \bar{\rho}\right)$ are trivial and obstructions to deforming can be detected locally.

- Once and for all choose for each $v \in S_{1}$ local deformations of $\left.\rho_{1}\right|_{G_{v}}$ to $\mathrm{GL}_{2}\left(\mathbb{Z}_{\ell}\right)$. For $v=\ell$ we choose a potentially semistable deformation. See [R3] for a proof that these local deformations exist.

- Since all local $\mathrm{GL}_{2}\left(\mathbb{F}_{\ell}\right)$ representations admit deformations to $\mathrm{GL}_{2}\left(\mathbb{Z} / \ell^{2} \mathbb{Z}\right)$ (see above) and global obstructions to deforming to $\mathrm{GL}_{2}\left(\mathbb{Z} / \ell^{2} \mathbb{Z}\right)$ can be detected locally, we can deform $\bar{\rho}$ to $\rho_{2}: \operatorname{Gal}(\overline{\mathbb{Q}} / \mathbb{Q}) \rightarrow \mathrm{GL}_{2}\left(\mathbb{Z} / \ell^{2} \mathbb{Z}\right)$ with $\operatorname{det}\left(\rho_{2}\right)=\varepsilon$.

- Choose a finite non-empty set $R_{1}$ of all primes unramified in $\rho_{2}$ beneath some bound, say $b_{1}$. Fix an element $r_{1} \in R_{1}$. The actual value of $b_{1}$ (and our later bounds $b_{2}, b_{3}, \ldots$ ) is not important. We will only require $b_{m} \rightarrow \infty$.

Once and for all, for each prime $r \in R_{1} \backslash\left\{r_{1}\right\}$ we choose an element in $W\left(\mathbb{F}_{\ell}\right)$ that will be the trace of Frobenius of $r$ in our eventual characteristiczero representation. For $r_{1}$ we choose an element in $W\left(\mathbb{F}_{\ell^{2}}\right) \backslash W\left(\mathbb{F}_{\ell}\right)$. For all $r \in R_{1}$ these elements are chosen congruent to $\operatorname{tr}\left(\rho_{2}\left(\sigma_{r}\right)\right) \bmod \ell$.

Recall all determinants of all deformations will be the cyclotomic character. Thus for any $r \in R_{1}$ the characteristic polynomial of $\sigma_{r}$ in our eventual characteristic-zero representation has already been determined and has coefficients in $W\left(\mathbb{F}_{\ell^{2}}\right) \subset \overline{\mathbb{Q}}_{\ell}$.

For $r \in R_{1}$, the trace of Frobenius at $r$ in $\rho_{2}$ may not be the mod $\ell^{2}$ reduction of the element characteristic-zero element just chosen. Similarly, for $v \in S_{1},\left.\rho_{2}\right|_{G_{v}}$ may not have mod $\ell^{2}$ reduction of the local representation chosen above. We are in a situation where we can apply Lemma 4.7 and Proposition 4.8.

- We use Lemma 4.7 to find a finite set of primes $Q_{1}$ and an element $g_{2} \in$ $H^{1}\left(G_{S_{1} \cup Q_{1}}, \operatorname{Ad}^{0} \bar{\rho}\right) \otimes_{\mathbb{F}_{\ell}} \mathbb{F}_{\ell^{2}}$ such that for all $r \in R_{2}$ we have $\operatorname{tr}\left(\left(I+\ell g_{2}\right) \rho_{2}\left(\sigma_{r}\right)\right)$ is the mod $\ell^{2}$ reduction of characteristic-zero number chosen above and so that at the places of $S_{1}$ the local representations 
are the mod $\ell^{2}$ reduction of the previously chosen characteristic-zero local representatons. Thus there are no obstructions to deforming to $\bmod \ell^{3}$ at primes of $S_{1}$. The representation $\left(I+\ell g_{2}\right) \rho_{2}$ may be ramified at primes in the set $Q_{1}$ of Lemma 4.7. There may be obstructions at primes of $Q_{1}$ to deforming to $\bmod \ell^{3}$.

- One by one we will remove obstructions at primes of $Q_{1}$ to deforming to $\bmod \ell^{3}$. Let $U_{1}=\cup \tilde{T}_{k}$ of Proposition 4.8. By that proposition we can choose an element $h_{2} \in H^{1}\left(G_{S_{1} \cup Q_{1} \cup U_{1}}, \operatorname{Ad}^{0} \bar{\rho}\right) \otimes_{\mathbb{F}_{\ell}} \mathbb{F}_{\ell^{2}}$ with $h_{2}=\sum_{i=1}^{n} \alpha_{i} f_{i}$ where the $f_{i}$ are as in Proposition 4.8 and the $\alpha_{i} \in \mathbb{F}_{\ell^{2}}$ such that

- For $v \in R_{1}$ we have that $\left.\operatorname{tr}\left(I+\ell\left(g_{2}+h_{2}\right)\right) \rho_{2}\right)\left(\sigma_{v}\right)$ is the $\bmod \ell^{2}$ reduction of the previously chosen characteristic-zero value,

- For $v \in S_{1}$ we have that $\left.\left(I+\ell\left(g_{2}+h_{2}\right)\right) \rho_{2}\right)\left(\sigma_{v}\right)$ is the $\bmod \ell^{2}$ reduction of the previously chosen characteristic-zero local at $v$ representation,

- for $v \in U_{1}$ there are no obstructions to deforming $\left.\left(I+\ell\left(g_{2}+h_{2}\right)\right) \rho_{2}\right)\left.\right|_{G_{v}}$ to characteristic-zero. (This uses the first bulleted item of Proposition 4.8.

Let $S_{2}=S_{1} \cup Q_{1} \cup U_{1}$ contain the set of ramified primes of this new deformation. For each $v \in S_{2} \backslash S_{1}$ once and for all choose local deformations of (our new) $\left.\rho_{2}\right|_{G_{v}}$ to $\mathrm{GL}_{2}\left(W\left(\mathbb{F}_{\ell^{2}}\right)\right.$ ). (We have no control over whether $\left.\rho_{2}\right|_{G_{v}}$ is ramified. If $\left.\rho_{2}\right|_{G_{v}}$ is unramified, we may choose our characteristic zero deformation to be either unramified or ramified as we please.)

- Deform $\rho_{2}$ to $\rho_{3}: G_{S_{2}} \rightarrow \mathrm{GL}_{2}\left(W\left(\mathbb{F}_{\ell^{2}}\right) / \ell^{3} W\left(\mathbb{F}_{\ell^{2}}\right)\right)$. Note that at places in $S_{2} \cup R_{1}$ we cannot guarantee that $\rho_{3}$ has the desired mod $\ell^{3}$ local behavior.

- We repeat this procedure. At the $m$ th stage we will have obtained a representation

$$
\rho_{m}: G_{S_{m-1}} \rightarrow \mathrm{GL}_{2}\left(W\left(\mathbb{F}_{\ell^{2^{m-2}}}\right) / \ell^{m} W\left(\mathbb{F}_{\ell^{2 m-2}}\right)\right)
$$

that may not agree with our previously chosen local characteristic-zero data at primes of $S_{m-1} \cup R_{m-2}$. Let $R_{m-1}$ be all primes unramified in $\rho_{m}$ beneath some bound $b_{m}$ such that $R_{m-1} \backslash R_{m-2}$ contains at least one element $r_{m-1}$. Once and for all, for each prime $r \in R_{m-1} \backslash\left(R_{m-1} \cup\left\{r_{m-1}\right\}\right)$ we choose an element in $W\left(\mathbb{F}_{\ell^{2}}{ }^{m-2}\right)$ that will be the trace of Frobenius of $r$ in our eventual characteristic-zero representation. For $r_{m-1}$ we choose an element in $W\left(\mathbb{F}_{\ell^{2}}\right) \backslash W\left(\mathbb{F}_{\ell^{2}-2}\right)$. For all $r \in R_{m-1} \backslash R_{m-2}$ these elements are chosen congruent to $\operatorname{tr}\left(\rho_{m}\left(\sigma_{r}\right)\right) \bmod \ell^{m-1}$. We then use Lemma 4.7 as before to adjust $\rho_{m}$ by an element of $H^{1}\left(\operatorname{Gal}(\overline{\mathbb{Q}} / \mathbb{Q}), \operatorname{Ad}^{0} \bar{\rho}\right) \otimes_{\mathbb{F}_{\ell}} \mathbb{F}_{\ell^{2}}{ }^{\mathrm{m}-1}$ so that our new representation agrees with the previously chosen local characteristic-zero data at primes of $S_{m-1} \cup R_{m-1}$. This may introduce ramification at new primes in a set $Q_{m-1}$. We use Proposition 4.8 to remove the obstructions at these new primes. Further new primes of ramification in a set $U_{m-1}$ may be introduced by this last procedure, but there will be no local obstructions to deforming at these new primes. Let $S_{m}=S_{m-1} \cup Q_{m-1} \cup U_{m-1}$. For each $v \in S_{m} \backslash S_{m-1}$ once and for all 
choose local deformations of (our new) $\left.\rho_{m}\right|_{G_{v}}$ to $\mathrm{GL}_{2}\left(W\left(\mathbb{F}_{\ell^{2 m-1}}\right)\right)$. We may continue the inductive deformation process.

The inverse limit of the compatible system of $\bmod \ell^{m}$ representations is valued in $\mathrm{GL}_{2}\left(W\left(\overline{\mathbb{F}}_{\ell}\right)\right)$. By the choices of the traces of the Frobenii of $r_{i}$ this representation is not valued in $\mathrm{GL}_{2}\left(W\left(\mathbb{F}_{\ell^{d}}\right)\right)$ for any $d$. Since $b_{m} \rightarrow \infty$, applying Theorem 2.5 and the Baire Category theorem, we see that our construction yields a representation with the properties asserted in Theorem 4.1.

\section{Acknowledgements}

We would like to thank Dipendra Prasad and Shankar Sen for some helpful correspondence. We also thank Brian Conrad for spotting a gap in an earlier proof of the results in Section 3, and J-P. Serre for helpful comments on the manuscript. Finally we thank the anonymous referee for numerous helpful suggestions.

\section{References}

[EGA IV] A. Grothendieck, Éléments de géométrie algébrique. IV. Étude locale des schémas et des morphismes de schémas. I. Inst. Hautes Études Sci. Publ. Math. 20 (1964) 5-259.

[Kh] C. Khare, Limits of residually irreducible p-adic Galois representations, Proc. Amer. Math. Soc. 131 (2003) 1999-2006.

[Kh-Raj] C. Khare, C.S. Rajan, The density of ramified primes in semisimple p-adic Galois representations, Internat. Math. Res. Notices, no. 12, (2001) 601-607.

[KLR] C. Khare, M. Larsen, and R. Ramakrishna, Constructing semisimple p-adic Galois representations with prescribed properties, to appear in Amer. J. Math.

[M] B. Mazur, An introduction to the deformation theory of Galois representations, Modular forms and Fermat's last theorem, edited by Gary Cornell, Joseph H. Silverman and Glenn Stevens. Springer-Verlag, New York, 1997.

[R1] R. Ramakrishna, Lifting Galois representations, Invent. Math. 138, no. 3, (1999) $537-562$.

[R2] _ Infinitely ramified representations, Ann. of Math. 151 (2000) 793-815.

[R3] Deforming Galois representations and the conjectures of Serre and Fontaine-Mazur, Ann. of Mathematics 156 (2002), 115-154.

[S1] J-P. Serre, Abelian -adic Representations and Elliptic curves, W.A. Benjamin 1968.

[S2] Quelques applications du théorème de densité de Chebotarev, Inst. Hautes Études Sci. Publ. Math. No. 54 (1981), 323-401. Collected Works, 3, 563-641.

[T] R. Taylor, Galois representations associated to Siegel modular forms of low weight, Duke Math. Journal 63 (1991), 281-332.

155 S 1400 E, Dept of Math, Univ of Utah, Salt Lake City, UT 84112 and School of Mathematics, TiFR, Homi Bhabha Road, Mumbai 400 005, India.

E-mail address: shekhar@math.utah.edu, shekhar@math.tifr.res.in

Department of Mathematics, Indiana University, Bloomington, IN 47405.

E-mail address: larsen@math.indiana.edu

Department of Mathematics, Cornell University, Malott Hall, Ithaca, Ny 14853.

E-mail address: ravi@math.cornell.edu 